\title{
Clinical characteristics of 60 discharged cases of 2019 novel coronavirus-infected pneumonia in Taizhou, China
}

\author{
Yongpo Jiang ${ }^{1}$, Susu He ${ }^{2}$, Chao Zhang ${ }^{1}$, Xiaodan Wang ${ }^{3}, \mathrm{Xi} \mathrm{Chen}^{2}$, Yingying Jin ${ }^{1}, \mathrm{Zebao} \mathrm{He}^{4}$, Minjie Cai ${ }^{1}$, \\ Zongmei Lin ${ }^{4}$, Lingjun Ying ${ }^{4}$, Jianli Mou ${ }^{4}$, Haihong Zhao ${ }^{4}$, Ronghai Lin ${ }^{1}$, Sheng Zhang ${ }^{1}$, Xiaomai $\mathrm{Wu}^{2}$, \\ Haixiao Chen ${ }^{5}$, Dongqing $\mathrm{Lv}^{2}$
}

${ }^{1}$ Department of Critical Care Medicine, Taizhou Hospital of Zhejiang Province, Wenzhou Medical University, Taizhou 317000, China; ${ }^{2}$ Department of Respiratory Medicine, Taizhou Hospital of Zhejiang Province, Wenzhou Medical University, Taizhou 317000, China; ${ }^{3}$ Department of Anesthesiology, Taizhou Hospital of Zhejiang Province, Wenzhou Medical University, Taizhou 317000, China; ${ }^{4}$ Department of Infectious Diseases, Taizhou Enze Medical Center (Group) Enze Hospital, Taizhou Public Health Center, Taizhou 318050, China; ${ }^{5}$ Department of orthopedics, Taizhou Hospital of Zhejiang Province, Wenzhou Medical University, Taizhou 317000, China

Contributions: (I) Conception and design: Y Jiang, D Lv; (II) Administrative support: X Wu, H Chen; (III) Provision of study materials: C Zhang, Z He; (IV) Collection and assembly of data: S He, X Wang, X Chen, Y Jin, M Cai, Z Lin, L Ying, J Mou, H Zhao; (V) Data analysis and interpretation: R Lin, S Zhang; (VI) Manuscript writing: All authors; (VII) Final approval of manuscript: All authors.

Correspondence to: Professor Ronghai Lin; Professor Haixiao Chen; Professor Dongqing Lv, MD. Taizhou Hospital of Zhejiang Province, Wenzhou Medical University, No. 150, Ximen Street, Taizhou 317000, China. Email: linrh@enzemed.com; chenhx@enzemed.com; lvdq@enzemed.com.

Background: The number of patients with pneumonia stemming from the 2019 novel coronavirus (COVID-19) infection has increased rapidly. However, the clinical characteristics of discharged patients remain little known. Here, we attempt to describe the clinical characteristics and treatment experiences of discharged cases from Taizhou, China.

Methods: A total of 60 patients with COVID-19-infected pneumonia who were discharged from Taizhou Enze Medical Center (Group), from January 31, 2020, to February 16, 2020, were included in the analysis. The discharge criteria were based on the New Coronavirus Pneumonia Prevention and Control Program (Fifth Edition, China).

Results: Of the 60 patients, the median age was 41 years, and $58.3 \%$ were male. Only $13.3 \%$ of patients were identified as having severe novel coronavirus pneumonia. All patients received combined antiviral treatment on admission, including $\beta$-interferon, lopinavir/tonavir, Abidol and oseltamivir. All patients with severe conditions received gamma globulin and hormone therapy. No patients had endotracheal intubation or died. The median duration from symptom onset to hospitalization was 3 (range, 0-15) days. The median duration of COVID-19 shedding was 14 (range, 5-26) days, and the median duration of hospital stay was 15 (range, 7-23) days.

Conclusions: Early therapy and comprehensive therapy are key to the outcome for patients with COVID19-infected pneumonia, especially for those with severe pneumonia.

Trial registration number: ChiCTR2000029866.

Keywords: 2019 novel coronavirus (COVID-19); 2019 novel coronavirus-infection pneumonia (COVID-19infection pneumonia; early treatment; antiviral

Submitted Mar 10, 2020. Accepted for publication Mar 30, 2020.

doi: 10.21037/atm.2020.04.20

View this article at: http://dx.doi.org/10.21037/atm.2020.04.20 


\section{Introduction}

The 2019 novel coronavirus-infected pneumonia (NCIP) is a viral infectious disease that began in Wuhan, China and is now a global concern $(1,2)$. Scientists have revealed a novel $\beta$-coronavirus from patients with NCIP, which is currently named COVID-19 (3). COVID-19 is a single-stranded RNA $\beta$-coronavirus with high infectivity (3). Similar to those of severe acute respiratory syndrome (SARS) and Middle East respiratory syndrome (MERS), the COVID-19 genome encodes nonstructural proteins, structural proteins, and accessory proteins $(3,4)$. A familial cluster of NCIP has indicated person-to-person transmission (5), and COVID-19-infected patients are highly person-to-person infectious (6,7). COVID-19 is prevalent around the world today. Preventing COVID-19 from spreading is extremely difficult. Some severe cases have rapidly progressed to acute respiratory syndrome (ARDS) or even died (8). How to prevent and treat is the focus.

Effective antiviral therapy is very important for patients with COVID-19. Unfortunately, for patients with COVID-19, there are currently no specific antiviral drugs available, and supportive care is the main strategy (9). Therefore, effective antiviral drugs against COVID-19 are urgently needed during the COVID-19 outbreak. Corticosteroid treatment in patients with COVID-19 is controversial, which is a double-edged sword. The best of time and dosage of corticosteroid treat in NICP is relied on doctors' clinical experience. Thence, we conducted a retrospective, single-center analysis to reveal the clinical characteristics and treatment experience of 60 discharged patients to provide a clinical therapy reference for patients with NCIP.

\section{Methods}

\section{Study design and patients}

This study is a retrospective, observational, single-center study. The research was registered in the Chinese Clinical Trial Registry (ChiCTR2000029866). Ethics approval was granted by Hospital Ethics Committee of Enze Hospital of Taizhou Enze Medical Center (Group). Patients from Enze Hospital, Taizhou Enze Medical Center (Group), from January 31, 2020, to February 16, 2020, were included in the analysis. Written informed consent was obtained from all participants before inclusion.

\section{Data collection}

All data were extracted from electronic medical records, and included epidemiological characteristics (such as recent exposure history), clinical symptoms and signs, and laboratory findings. All patients were from Enze Hospital, Taizhou Enze Medical Center (Group), which includes the of Taizhou Public Health Center. Laboratory-confirmed cases with COVID-19-related pneumonia who improved and were discharged were consecutively included.

\section{Diagnostic criteria}

(I) Laboratory reverse transcription polymerase chain reaction (RT-PCR) was used to confirm COVID-19 infection, while (II) chest imaging was used to confirm lung involvement.

\section{Discharge criteria}

The criteria for patient discharge was as follows: (I) temperature returning to normal for at least 3 days; (II) significant improvement in respiratory symptoms; (III) inflammatory lung imaging showing significant absorption; (IV) two consecutive negative results of respiratory pathogen nucleic acid detection (sampling time interval of at least 1 day) (10).

\section{Diagnostic criteria for severe patients}

Patients were considered severe if they met any of the following criteria: respiratory distress [respiratory rate (RR) $>30$ breaths/min at rest], mean oxygen saturation $\leq 93 \%$; arterial oxygen pressure/oxygen concentration $\left(\mathrm{PaO}_{2} / \mathrm{FiO}_{2}\right)$ $\leq 300 \mathrm{mmHg}(10)$.

\section{RT-PCR for COVID-19 RNA}

Laboratory confirmation of COVID-19 was conducted in the Taizhou Municipal Center for Disease Control and Prevention and Health Clinic for Enze Precision Medicine. The RT-PCR assay was conducted in accordance with the protocol established by the World Health Organization (11). 
Firstly, RNA was extracted from $300 \mu \mathrm{L}$ kinds of clinical samples using the SARS-CoV-2 RNA Kit (Zhijiang, Z-RR0479-02-50). Then the targeting SARS-CoV-2 open reading frame1ab gene was amplification determined in an ABI 7500 machine as follows: $45^{\circ} \mathrm{C}$ for $10 \mathrm{~min}, 95^{\circ} \mathrm{C}$ for $3 \mathrm{~min}$ followed by 45 cycles consisting of $95^{\circ} \mathrm{C}$ for $15 \mathrm{~s}, 58{ }^{\circ} \mathrm{C}$ for $30 \mathrm{~s}$ and finally a default melting curve step.

\section{Detection of lymphokines}

A flow cytometric bead array (CBA) was used as a rapid determination tool for Th1/Th2 cytokines, including IL-2, IL-4, IL-6, IL-10, TNF, and IFN- $\gamma$. The quantification of the 6 cytokines was evaluated by a FACSAria II Cell Sorter (BD, CA, USA) with a human Th1/Th2 subpopulation detection kit (CEGER, Zhejiang, China). The data acquired from the FACSAria II were analyzed with BD FCAP Array v.3.0.1 software. In total, 6 standard curves were obtained from 1 set of calibrators, and 6 results were obtained from each test sample. The maximum and minimum limits of the 6 detected cytokines were 1.0 and $5,000 \mathrm{pg} / \mathrm{mL}$, respectively. Biochemical measurement was performed by a Siemens Advia 2400 (Siemens, BER, Germany).

\section{Statistical methods}

Data are expressed as the mean \pm standard deviation (SD), mean \pm standard error $(\mathrm{SE})$, median with range, or percent with number of patients. Normality was evaluated by using the Shapiro-Wilk test (P value $>0.05)$. Comparisons between groups of continuous variables that were normally distributed were carried out using Student's $t$-test or a corrected $t$-test. Comparisons of other scenarios were performed using the Mann-Whitney $U$ test. Comparisons of categorical variables were performed using the $\chi^{2}$ test or Fisher's exact test. $\mathrm{P}$ values $<0.05$ were considered significant. All statistical analyses were performed with SPSS 22.0.

\section{Results}

Among the 60 patients, $13.3 \%(n=8)$ had severe novel coronavirus pneumonia, $75 \%$ had visited Wuhan, and 25\% had contact with people from Wuhan. The median age was 41 (range, 12-74) years, and 58.3\% were male. The most common diseases in their personal medical histories included hypertension (8.3\%) and diabetes (1.7\%). Fever (71.7\%) and cough (56.7\%) were the most common symptoms. Diarrhea was uncommon (Table 1). The time from onset to hospitalization was 3 (range, 0-15) days, and diagnosis occurred 5.0 days after onset (range, $0-15$ ). The time from onset to the beginning of antiviral therapy was 6.0 (range, 1.0-18.0) days (Table 1).

On admission, the white blood cell count did not increase in any of the patients, and the patients had obvious lymphopenia. The severe cases had more prominent laboratory abnormalities (e.g., potassium, phosphorus, $\mathrm{PaCO}_{2}, \mathrm{PaO}_{2} / \mathrm{FiO}_{2}$ ) than the non-severe cases (Table 2). Significantly higher levels of several inflammatory factors, including IL-6, IL-10, and TFN- $\gamma$, were observed, although the levels of the inflammatory factors IL-2, IL4 , and TNF- $\alpha$ were not increased significantly. When the respiratory pathogen nucleic acid test was negative, the levels of the inflammatory factors decreased (Figure 1).

All patients were given antiviral treatment: $\beta$-interferon + lopinavir/tonavir, $\beta$-interferon + lopinavir/tonavir + Abidol, and $\beta$-interferon + lopinavir/tonavir + oseltamivir therapy were initiated in $70 \%, 25 \%$, and $5 \%$ of patients, respectively. In addition, all severe patients were treated with glucocorticoids and gamma globulin. The median duration of COVID-19 shedding was 14 days, and the median duration of hospital stay was 15 days (Table 3).

\section{Discussion}

Early isolation and early treatment are very important for patients with COVID-19-infected pneumonia (NCIP). In this research, the median duration from symptom onset to hospitalization was 3 days, and the median duration from symptom onset to diagnosis confirmation was 5 days, which was relatively short. Most of the patients included in our study had a history of exposure in Wuhan, and the local government of Taizhou implemented medical isolation and observation at an early stage. After the onset of initial symptoms, such as fever and coughing, routine blood examination and chest computed tomography (CT) were immediately performed. If suspected patients were found, nucleic acid tests were conducted immediately to enable early diagnosis and treatment.

At present, glucocorticoid treatment for NCIP is controversial (9). High-dose glucocorticoid therapy is not recommended in SARS (12), and current evidence suggests that high-dose corticosteroid treatment does not improve the prognosis of patients with ARDS (13). Moreover, corticosteroid treatment is not routinely recommended for SARS-CoV-2 pneumonia (14,15). However, we observed 
Table 1 Baseline characteristics of 60 patients with COVID-19 on admission

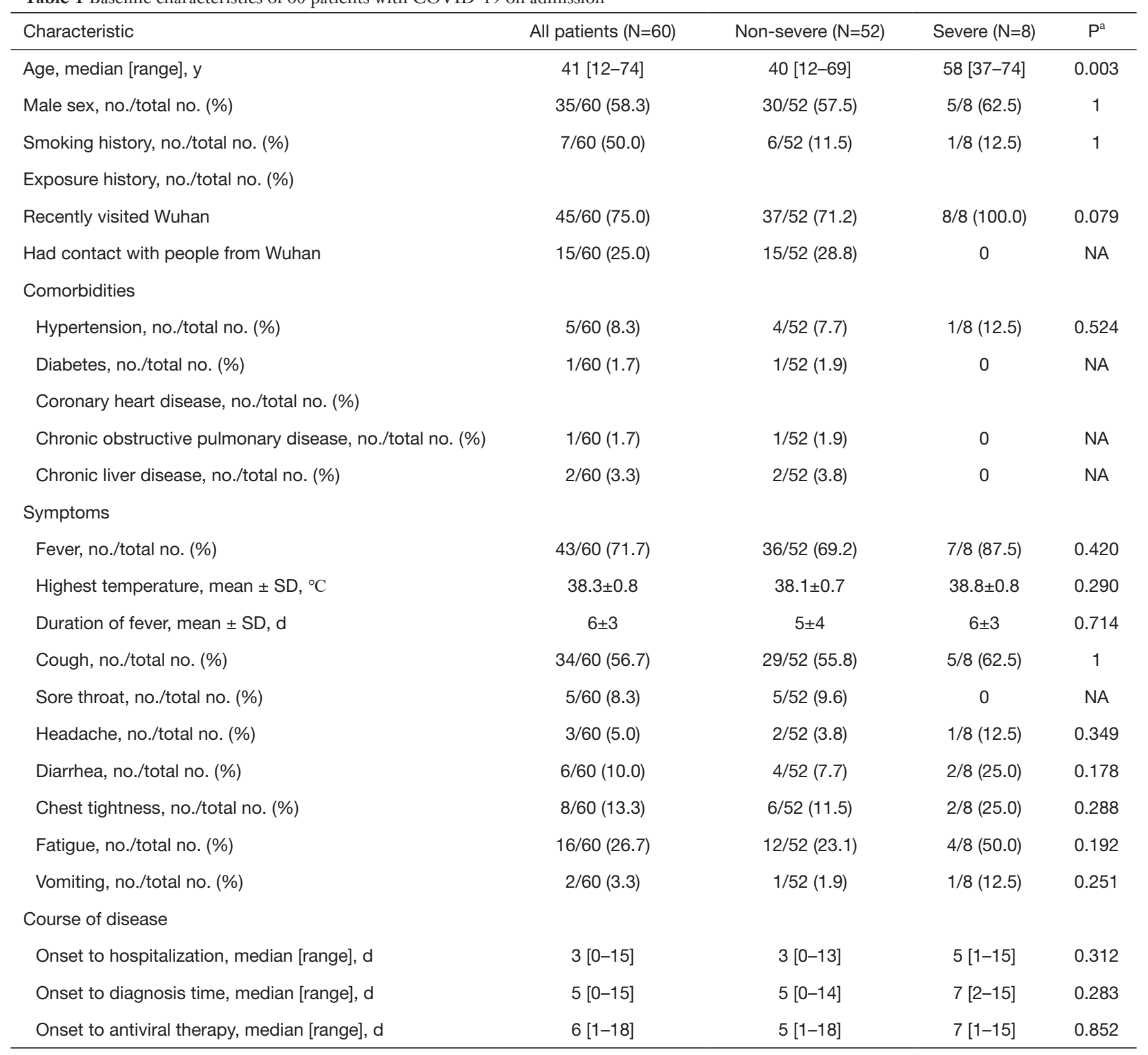

a, Fisher's exact test, Student's $t$-test, and Mann-Whitney U test. COVID-19, 2019 novel coronavirus.

elevated levels of inflammatory markers, such as IL-6 and TFN- $\gamma$, in our patients (Figure 1). Cytokine storms and immunopathology are major causes of ARDS in patients with COVID-19-infected pneumonia (16). The latest pathological findings, including pulmonary and peripheral blood results, suggest that timely and appropriate use of corticosteroids should be considered for severe patients to prevent ARDS development (17). In our center, when a patient's $\mathrm{PaO}_{2} / \mathrm{FiO}_{2}$ is less than $300 \mathrm{mmHg}$, radiological manifestations of lung injury are aggravated, or dyspnea occurs, hormone and gamma globulin therapy are performed (Figure 2). The period of hormone therapy is approximately 1 week, and that of gamma globulin therapy is approximately 3 to 5 days. Notably, none of the severe patients progressed to critical illness or died. Our team found that it is important to detect severe cases in 
Table 2 Laboratory items of 60 patients with COVID-19

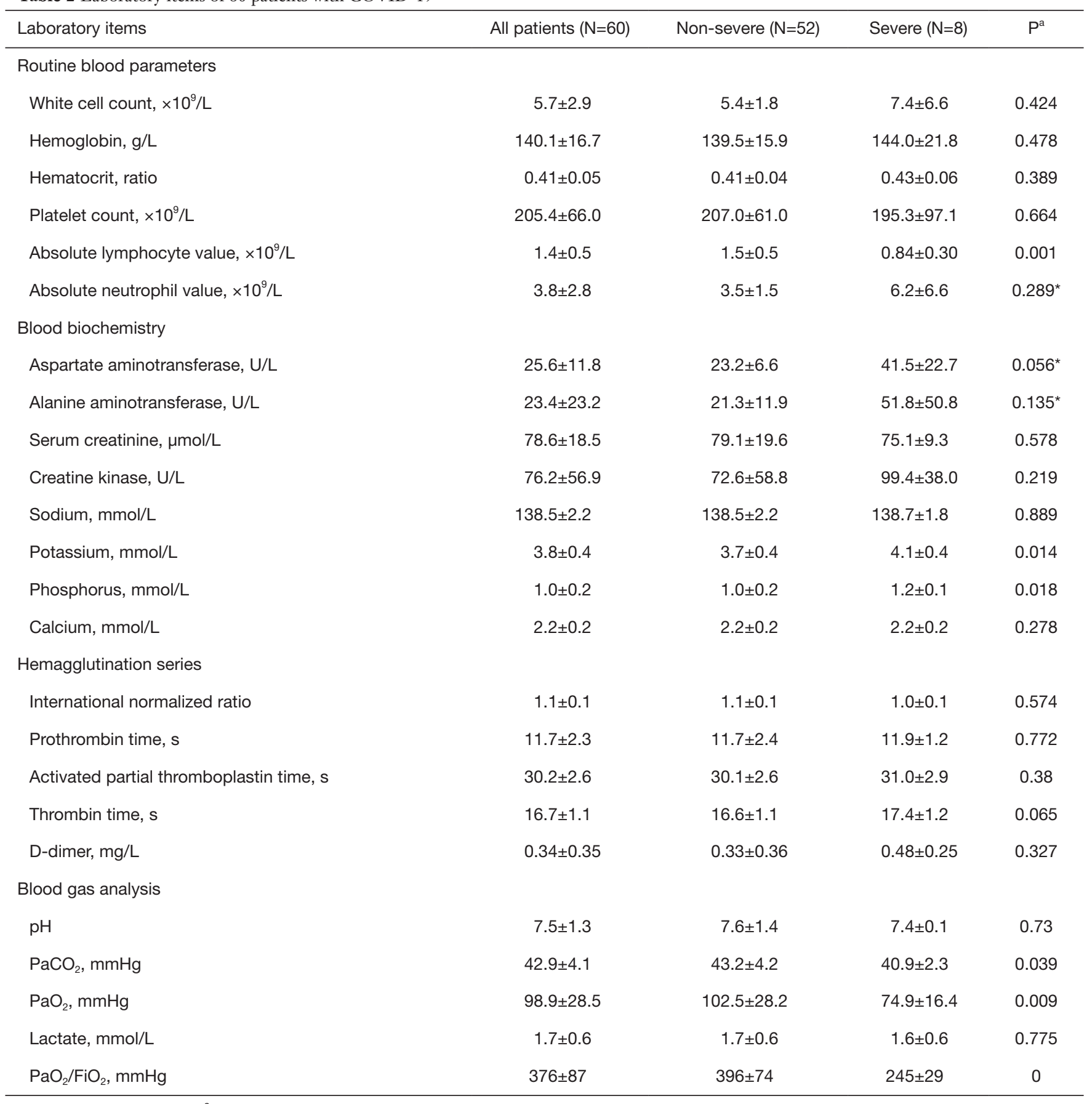

*, Mann-Whitney U test; ${ }^{a}$, student's $t$-test and corrected $t$-test. COVID-19, 2019 novel coronavirus. All data are expressed as mean \pm SD.

order to provide hormone and gamma globulin therapy in a timely manner. We look forward to the results of the glucocorticoid randomized controlled trial (RCT) (clinical trial registration number: ChiCTR2000029386).
In this study, all the patients were given antiviral treatment, which was usually a combination of 2 antiviral drugs, including $\beta$-interferon, lopinavir/tonavir, Abidol, and oseltamivir. The median duration of COVID-19 shedding 


\section{Page 6 of 8}

was 14 days. The effects of these antiviral drugs are still unclear, and further RCT studies are needed. All the patients required observation and isolation after discharge, and nucleic acid detection of feces and sputum specimens was performed 2 weeks after discharge. Some studies have found that COVID-19-infected patients' feces could harbor the virus for a long time (18), and it is currently uncertain whether fecal-oral transmission exists (18). Thus, we continued to monitor the patients carefully after their release.

Comprehensive measures are crucial for treating

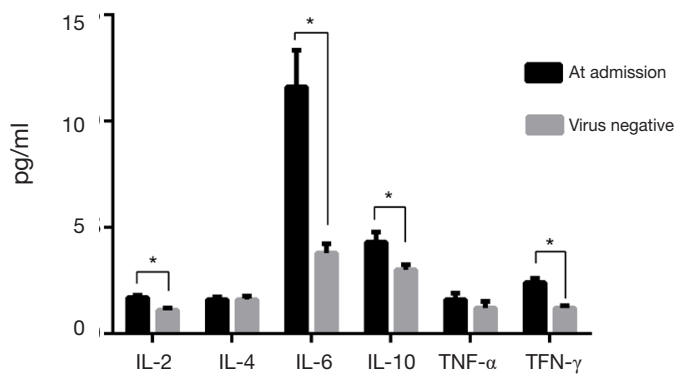

Figure 1 The levels of inflammatory factors, including IL-2, IL4, IL-6, IL-10, TNF- $\alpha$, and TFN- $\gamma$ (All data are expressed as Mean \pm SE), at the time of hospital admission and at the time of testing negative for the virus. Differences were considered to be statistically significant when $\mathrm{P}<0.05$.
Jiang et al. Discharged cases of COVID-19-infected pneumonia

NCIP. In our center, physicians from the Department of Intensive Care and the Department of Respiratory and Infectious Disease, combined with psychologists, physical therapists and Traditional Chinese Medicine (TCM) physicians, participated throughout the treatment of each patient with NCIP to ensure that the patients received comprehensive treatment.

In conclusion, we here described the clinical characteristics of discharged patients with NCIP and shared our management experience. Our results suggest that early therapy and comprehensive therapy are important in NCIP treatment, especially for critical patients with severe pneumonia.

\section{Acknowledgments}

We would like to thank all the physicians and nurses in the Taizhou Public Health Center.

Funding: This work was supported by The Science and Technology Project of Taizhou (no. 1902ky02, 1902ky27) and The Natural Science Foundation of Zhejiang Province (no. Y20H150029).

\section{Footnote}

Conflicts of Interest: All authors have completed the ICMJE uniform disclosure form (available at http://dx.doi.

Table 3 Treatments and clinical outcomes

\begin{tabular}{|c|c|c|c|c|}
\hline Treatments & All patients $(\mathrm{N}=60)$ & Non-severe $(\mathrm{N}=52)$ & Severe $(N=8)$ & $\mathrm{P}^{\mathrm{a}}$ \\
\hline$\beta$-interferon + lopinavir/tonavir, no./total no. [\%] & $42 / 60[70]$ & $39 / 52[80]$ & $3 / 8[38]$ & 0.045 \\
\hline$\beta$-interferon + lopinavir/tonavir + abidol, no./total no. [\%] & $15 / 60[25]$ & $10 / 52[19]$ & $5 / 8[63]$ & 0.019 \\
\hline$\beta$-interferon + lopinavir/tonavir + oseltamivir, no./total no. [\%] & $3 / 60[5]$ & $3 / 52[6]$ & 0 & NA \\
\hline Glucocorticoid, no./total no. [\%] & $9 / 60[15]$ & $1 / 52[2]$ & $8 / 8[100]$ & 0 \\
\hline Gamma globulin, no./total no. [\%] & $9 / 60[15]$ & 0 & $8 / 8[100]$ & NA \\
\hline Intravenous antibiotics, no./total no. [\%] & $6 / 60[10]$ & $5 / 52[10]$ & $1 / 8[1]$ & 1.000 \\
\hline \multicolumn{5}{|l|}{ Clinical outcomes } \\
\hline Hospital stay, median [range], d & 15 [7-23] & $15[7-23]$ & 14 [8-23] & 0.571 \\
\hline
\end{tabular}

\footnotetext{
a , Fisher test and Mann-Whitney U test.
} 


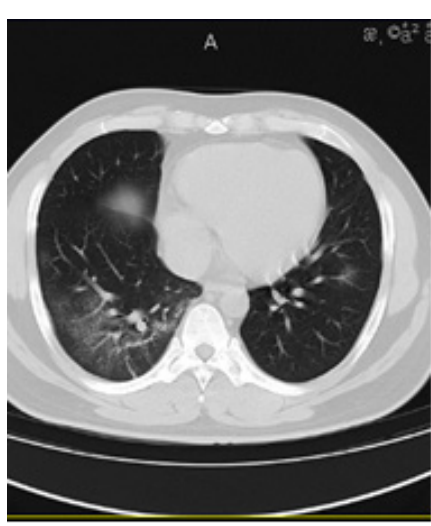

February 1

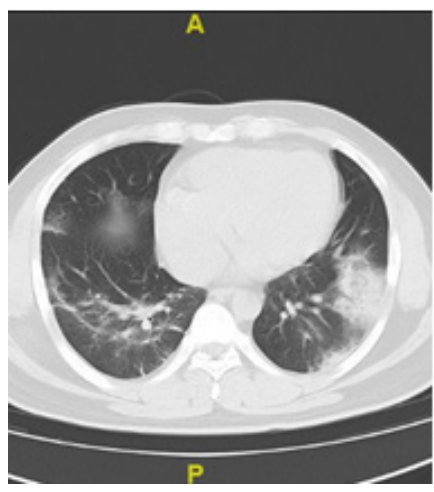

February 6

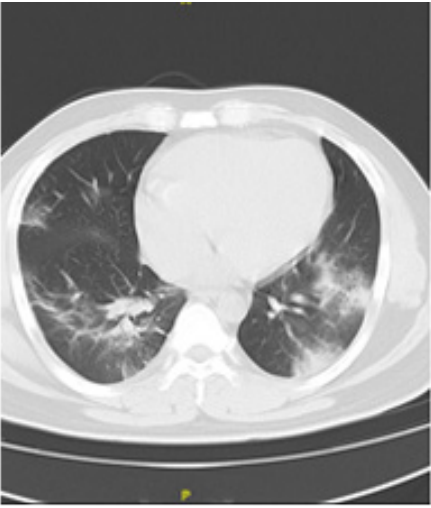

February 3

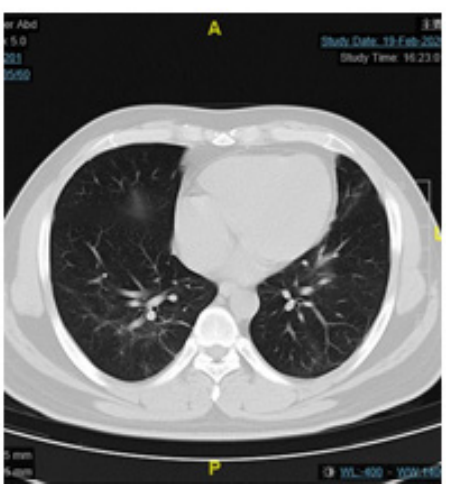

February 11

Figure 2 Chest computed tomography of a severe case who was admitted from February 1, 2020, to February 11, 2020. The patient was treated with glucocorticoids and gamma globules from February 3, to February 10.

org/10.21037/atm.2020.04.20). The authors have no conflicts of interest to declare.

Ethical Statement: The authors are accountable for all aspects of the work in ensuring that questions related to the accuracy or integrity of any part of the work are appropriately investigated and resolved. Ethics approval was granted by Hospital Ethics Committee of Enze Hospital of Taizhou Enze Medical Center (Group) (No. K20200204). Written informed consent was obtained from all participants before inclusion.

Open Access Statement: This is an Open Access article distributed in accordance with the Creative Commons Attribution-NonCommercial-NoDerivs 4.0 International License (CC BY-NC-ND 4.0), which permits the noncommercial replication and distribution of the article with the strict proviso that no changes or edits are made and the original work is properly cited (including links to both the formal publication through the relevant DOI and the license). See: https://creativecommons.org/licenses/by-nc-nd/4.0/.

\section{References}

1. Wang $\mathrm{D}, \mathrm{Hu} \mathrm{B}, \mathrm{Hu} \mathrm{C}$, et al. Clinical characteristics of 138 hospitalized patients with 2019 novel Coronavirus-infected pneumonia in Wuhan, China. JAMA 2020. [Epub ahead of print].

2. Huang C, Wang $\mathrm{Y}, \mathrm{Li}$ X, et al. Clinical features of patients infected with 2019 novel Coronavirus in Wuhan, China. Lancet 2020;395:497-506.

3. Lu R, Zhao X, Li J, et al. Genomic characterisation and epidemiology of 2019 novel Coronavirus: implications for virus origins and receptor binding. Lancet 
2020;395:565-74.

4. Zhu N, Zhang D, Wang W, et al. A novel Coronavirus from patients with pneumonia in China, 2019. N Engl J Med 2020;382:727-33.

5. Chan JF, Yuan S, Kok KH, et al. A familial cluster of pneumonia associated with the 2019 novel Coronavirus indicating person-to-person transmission: a study of a family cluster. Lancet 2020;395:514-23.

6. Phan LT, Nguyen TV, Luong QC, et al. Importation and human-to-human transmission of a novel Coronavirus in Vietnam. N Engl J Med 2020;382:872-4.

7. Li Q, Guan X, Wu P, et al. Early transmission dynamics in Wuhan, China, of novel Coronavirus-infected pneumonia. N Engl J Med 2020;382:1199-207.

8. Guan WJ, Ni ZY, Hu Y, et al. Clinical characteristics of 2019 novel Coronavirus infection in China. medRxiv 2020. doi: 10.1056/NEJMoa2002032.

9. Lu H. Drug treatment options for the 2019-new Coronavirus (2019-nCoV). Biosci Trends 2020;14:69-71.

10. National Health Commission of China. The new Coronavirus pneumonia prevention and control program. China: National Health Commission of China; 2020.

11. World Health Organization. Laboratory testing for 2019 novel Coronavirus (2019-nCoV) in suspected human cases. Geneva, Switzerland: World Health Organization; 2020.

Cite this article as: Jiang $\mathrm{Y}, \mathrm{He} \mathrm{S}$, Zhang C, Wang X, Chen X, Jin Y, He Z, Cai M, Lin Z, Ying L, Mou J, Zhao H, Lin R, Zhang S, Wu X, Chen H, Lv D. Clinical characteristics of 60 discharged cases of 2019 novel coronavirus-infected pneumonia in Taizhou, China. Ann Transl Med 2020;8(8):547. doi: 10.21037/atm.2020.04.20
12. Thompson BT, Ranieri VM. Steroids are part of rescue therapy in ARDS patients with refractory hypoxemia: no. Intensive Care Med 2016;42:921-3.

13. Kido T, Muramatsu K, Asakawa T, et al. The relationship between high-dose corticosteroid treatment and mortality in acute respiratory distress syndrome: a retrospective and observational study using a nationwide administrative database in Japan. BMC Pulm Med 2018;18:28.

14. Wu F, Zhao S, Yu B, et al. A new Coronavirus associated with human respiratory disease in China. Nature 2020;579:265-9.

15. Russell CD, Millar JE, Baillie JK. Clinical evidence does not support corticosteroid treatment for 2019-nCoV lung injury. Lancet 2020;395:473-5.

16. Channappanavar R, Perlman S. Pathogenic human Coronavirus infections: causes and consequences of cytokine storm and immunopathology. Semin Immunopathol 2017;39:529-39.

17. Xu Z, Shi L, Wang Y, et al. Pathological findings of COVID-19 associated with acute respiratory distress syndrome. Lancet Respir Med 2020;8:420-2.

18. Silverstein WK, Stroud L, Cleghorn GE, et al. First imported case of 2019 novel Coronavirus in Canada, presenting as mild pneumonia. Lancet 2020;395:734. 